\title{
An Optimal Backoff Time-Based Internetwork Interference Mitigation Method in Wireless Body Area Network
}

\author{
Zhijun Xie $\mathbb{D}^{1},{ }^{1}$ Baocheng Wang $\mathbb{D}^{1},{ }^{1}$ Jiancheng Yu $\left(\mathbb{D},{ }^{1}\right.$ Huanming Wu $\mathbb{D}^{1}$, \\ Guangyan Huang $\mathbb{D}^{2},{ }^{2}$ Roozbeh Zarei $\mathbb{D}^{2,3}$ and Jing $\mathrm{He} \mathbb{i D}^{4,5}$ \\ ${ }^{1}$ Department of Information Science and Engineering, Ningbo University, Ningbo, China \\ ${ }^{2}$ School of Information Technology, Deakin University, Melbourne, Australia \\ ${ }^{3}$ Ningbo Institute of Materials Technology \& Engineering, Chinese Academy of Sciences, Ningbo, China \\ ${ }^{4}$ Institute of Information Technology, Nanjing University of Finance and Economics, Jiangsu, Nanjing, China \\ ${ }^{5}$ Swinburne Data Science Research Institute, Swinburne University of Technology, Melbourne, Australia
}

Correspondence should be addressed to Zhijun Xie; xiezhijun@nbu.edu.cn, Baocheng Wang; wangbc1994@foxmail.com, and Jing He; lotusjing@gmail.com

Received 21 August 2019; Revised 29 November 2019; Accepted 8 January 2020; Published 2 March 2020

Academic Editor: Ghufran Ahmed

Copyright (c) 2020 Zhijun Xie et al. This is an open access article distributed under the Creative Commons Attribution License, which permits unrestricted use, distribution, and reproduction in any medium, provided the original work is properly cited.

\begin{abstract}
When multiple Wireless Body Area Networks (WBANs) are aggregated, the overlapping region of their communications will result in internetwork interference, which could impose severe impacts on the reliability of WBAN performance. Therefore, how to mitigate the internetwork interference becomes the key problem to be solved urgently in practical applications of WBAN. However, most of the current researches on internetwork interference focus on traditional cellular networks and large-scale wireless sensor networks. In this paper, an Optimal Backoff Time Interference Mitigation Algorithm (OBTIM) is proposed. This method performs rescheduling or channel switching when the performance of the WBANs falls below tolerance, utilizing the cell neighbour list established by the beacon method. Simulation results show that the proposed method improves the channel utilization and the network throughput, and in the meantime, reduces the collision probability and energy consumption, when compared with the contention-based beacon schedule scheme.
\end{abstract}

\section{Introduction}

Wireless Body Area Network (WBAN) is characterized by intensive and highly mobile deployments, especially in the warding areas or public places where the network density can become very high [1-4]. In WBANs, there is a lack of coordination among those random distributed and independent body area networks, which can easily lead to internetwork interference and seriously affect the reliability of the networks. The study of Natarajan et al. [5] showed that when the number of body area network reaches 8 or more, internetwork interference can result in the loss of data rate by over $35 \%$. Sun et al. [6] investigated the hospital WBAN interference problems in practical applications. Their survey discovered that even during the off-peak period, only $68.5 \%$ of the data transmission could meet reliability requirements, and with the increase of the node transmission power, the interference would become more serious. Therefore, internetwork interference could lead to loss of important data and cause false diagnoses, which can pose a serious threat to a patient's life and security $[7,8]$.

Recent years have seen many researchers working on network interference between the cellular network and the wireless sensor networks. For example, in the case of a cellular network, the interference cancellation technique of WLANs based on the Carrier Sense Multiple Access with Collision Avoidance (CSMA/CA) interference compensation mechanism is proposed and studied $[9,10]$. In view of the intercluster interference of the wireless sensor networks, an interference mitigation mechanism based on ad hoc timeslot 
reassignment is proposed [11]. However, due to greater deployment density, tighter energy constraints, and more critical reliability requirements, as well as the fact that network nodes lack global network information and their radio signals are highly dynamic, it is understood that the existing research results cannot be directly used in the body area networks $[12,13]$.

In this work, we design an Optimal Backoff Time Interference Mitigation Algorithm (OBTIM) for body area networks to address the aforementioned challenges. The method includes mechanisms of network interference detection, the asynchronous neighbour discovery method, and transmission rescheduling. The novelty of this method is that a method of backoff time optimization based on linear programming is proposed to increase the throughput and the asynchronous neighbour discovery method is proposed to establish a neighbour information table to help in the arrangement of dynamic retransmission and switching of channels according to the channel states. These actions will execute only if excessive interference is detected and will stop to continue regular data transmission after interference mitigation. By doing so, we minimize the time delay and energy consumption. The simulation results show that the OBTIM proposed in this paper outperforms the scheme based on beacon scheduling [14] and the scheme based on 802.15.4 [15], mainly in the following two aspects:

(1) The OBTIM has less time delay and collisions. The time delay will increase significantly in the competition-based beacon scheme [14] since multiple carrier interception is required for each transmission, and channel congestion will lead to a longer retreat time when the number of WBAN increases. In comparison, the OBTIM only performs rescheduling when interference is detected, which reduces the number of collisions and thus reduces the time delay

(2) Compared with those algorithms without optimization (the competition-based beacon scheme and the scheme based on 802.15.4), the OBTIM has less throughput and average energy consumption since the backoff time optimization method based on linear programming is used to find the backoff time to minimize the system throughput. We know that the scheduling operation includes a data transmission delay $T_{\text {delay, }}$ and $T_{\text {delay }}=T_{\text {work }}+T_{\text {backoff }}$, where $T_{\text {backoff }}$ is a backoff strategy deliberately set up by the system before sending a rescheduling beacon. Therefore, whether the backoff time $T_{\text {backoff }}$ is selected properly or not will affect throughput and average energy consumption

The advantages of the OBTIM are as follows: (1) short backoff time and low time delay; (2) the lowest probability of beacon conflict; (3) under different scenarios of WBAN number and channel number, the method in this paper has a better throughput; and (4) the average energy consumption of a successful transmission of a packet is significantly lower than the other two schemes.
The rest of this paper is organized as follows. Section 2 provides an overview of recent research development in interference mitigation technologies in WBANs. Section 3 explains network modelling and our proposed method for interference problems. In Section 4, we derive related performance indicators and present simulation assumptions and methods. Section 5 concludes this paper by summarizing the results and findings.

\section{Related Works}

The existing interference mitigation strategies mainly include techniques and algorithms in a frequency domain, power control, and cooperative communication.

In the frequency domain, technologies such as frequency band allocation, frequency domain analysis, and modulation are used to realize interference mitigation. GonzálezValenzuela et al. [16] proposed a method based on Frequency Division Multiple Access (FDMA) to allocate different frequency bands for each WBAN in the initialization of the network. However, it proved to be difficult to dynamically reuse the frequency bands among WBANs in this method. Zou et al. [17] proposed a method for interfrequency band interference analysis and a network deployment design scheme for Chinese medical body domain networks. This method takes into consideration the autocorrelation of two delay samples to establish narrow-band interference detection and broadband interference mitigation. The limitation of the method is that its implementation demands the use of Quadrature Phase Shift Keying (OQPSK) modulation in the physical layer, often implying the need for hardware modifications.

Power control is a very important technology in multiple access wireless networks for interference suppression. However, the centralized power control scheme often used in mobile cellular networks is not suitable for the distributed and independent WBANs. Wu et al. [18] proposed a series of methods, such as an algorithm based on measuring every neighbour WBAN interference, noncooperative game theory, and a learning algorithm without regret. They are all used to choose the appropriate channel and transmit power. The main disadvantage of these methods is the high iterations (more than 20 times) required to calculate the channel and power to achieve the best value, and before that the values need to be frequently changed and set which in turn could lead to system instability [19]. Movassaghi et al. [20] presented an adaptive interference mitigation scheme for multiple coexisting wireless body area networks (WBANs) based on social interaction, but there is a loss of energy in the calculation. Roy et al. [21] proposed a 2-hop cost-based energy-efficient routing protocol for WBAN. However, the utilization and throughput of the channel are not high.

Cooperative communication is another technology that can effectively mitigate interference in dense WBAN deployment. The advantage of cooperative communication lies in spatial diversity. When the transmission distance between the source and the host nodes is large, or the radio condition becomes severe, the transmission reliability and energy efficiency can be improved significantly through cooperative 


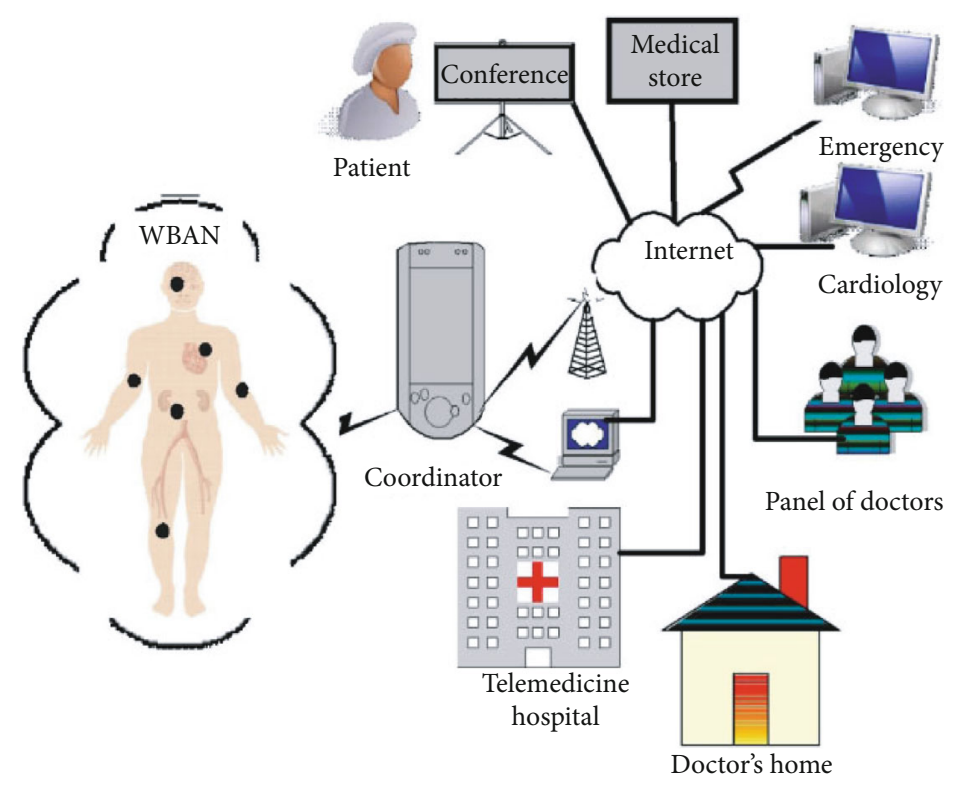

FIGURE 1: Health service system based on WBAN.

communication [22]. Le and Moh [23] proposed an interference-aware traffic-priority-based link scheduling (ITLS) algorithm to overcome inter-WBAN interference in densely deployed WBANs. However, it is not very effective in sparsely deployed node networks. Yu [24] researched and designed a relay selection strategy based on interruption probability and a transmission scheduling algorithm. The algorithm was further improved and optimized through dynamic timeslot allocation and the average channel gain forecast. However, this scheme did not consider overlapping communication areas caused by multiple WBANs.

Kim et al. [14] proposed a Time Division Multiple Access (TDMA) scheduling mechanism using distributed beacons, in order to avoid the collision when other body area networks try to access the same channel, and each beacon uses carrier sense before transmission, which may increase the energy overhead.

Considering the radio channel characteristics of body area networks, we adopt an optimal backoff time-based internetwork interference mitigation method. We take into consideration the periodicity of application data transmission in combined body area networks to reschedule or switch the channel in case of internetwork interference, hence improving the transmission reliability with great effect.

\section{System Modelling and Algorithm Design}

Figure 1 depicts a typical WBAN-based health service system. The interference of communication between the coordinator and the server can be solved by using the existing WAN technology and cellular technology. This paper mainly analyses the interference from the neighbour WBANs in the communication between the wireless sensor nodes, the node, and the coordinator, which is worn or implanted when multiple WBANs are present in the area [25-27]. In this paper, the neighbour table is established by beacons and the disturbance mitigation method based on the reservation is studied, and how to avoid a collision caused by an incomplete neighbour table is considered [28-31].

3.1. Problem Description. Figure 2 shows the superframe structure used in this work. The active data transmission stage is divided into competitive (Contention Access Period (CAP)) and noncompetitive periods (Contention-Free Period (CFP)). Firstly, the coordinator synchronizes each sensor node in the WBAN by broadcasting beacon B with dispatch information. The sensor nodes in the CAP communicate with other nodes through the time-gap CSMA/CA mechanism, and the CFP node uses TDMA to send data in the allocated timeslot. After the data transmission is complete, the node moves into sleep mode. The Beacon Interval (BI) indicates the length of a superframe, $\mathrm{BI}=$ aBaseSuperframeDuration $* 2^{\mathrm{BO}}, \mathrm{BO}$ is the 0 - 3 bit of the beacon frame, aBaseSuperframeDuration is a constant with a value of 960 symbols, and Superframe Duration (SD) represents the length of the active period; the initial timing parameter is $0.1 \mathrm{~s}$.

We consider a typical wireless body area network (e.g., the one shown in Figure 1) as network $i$. The scheduling information of network $i$ can be expressed as $\left(\mathrm{SD}^{i}, \mathrm{BI}^{i}, c^{i}, t^{i}\right)$, where $\mathrm{SD}^{i}$ and $\mathrm{BI}^{i}$ represent the data transmission duration and beacon interval in network $i$, respectively. $c^{i}$ represents the number of channels, and $t^{i}$ is the next transmission time. This information can be obtained by decoding the beacon transmission. When wireless body area network $j$ or $i$ moves close to each other, say, $\left\|t^{i}-t^{j}\right\| \leq \mathrm{SD}^{j}$, communication interference will occur. This is named internetwork interference. 


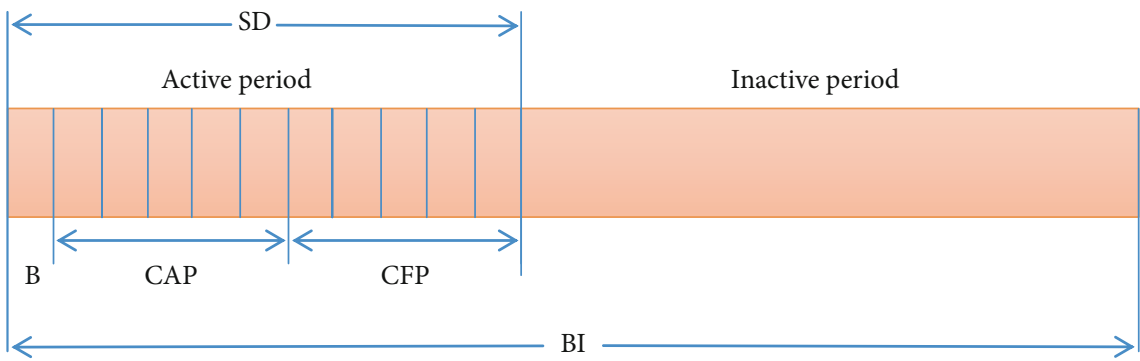

Figure 2: Superframe model with the beacon.

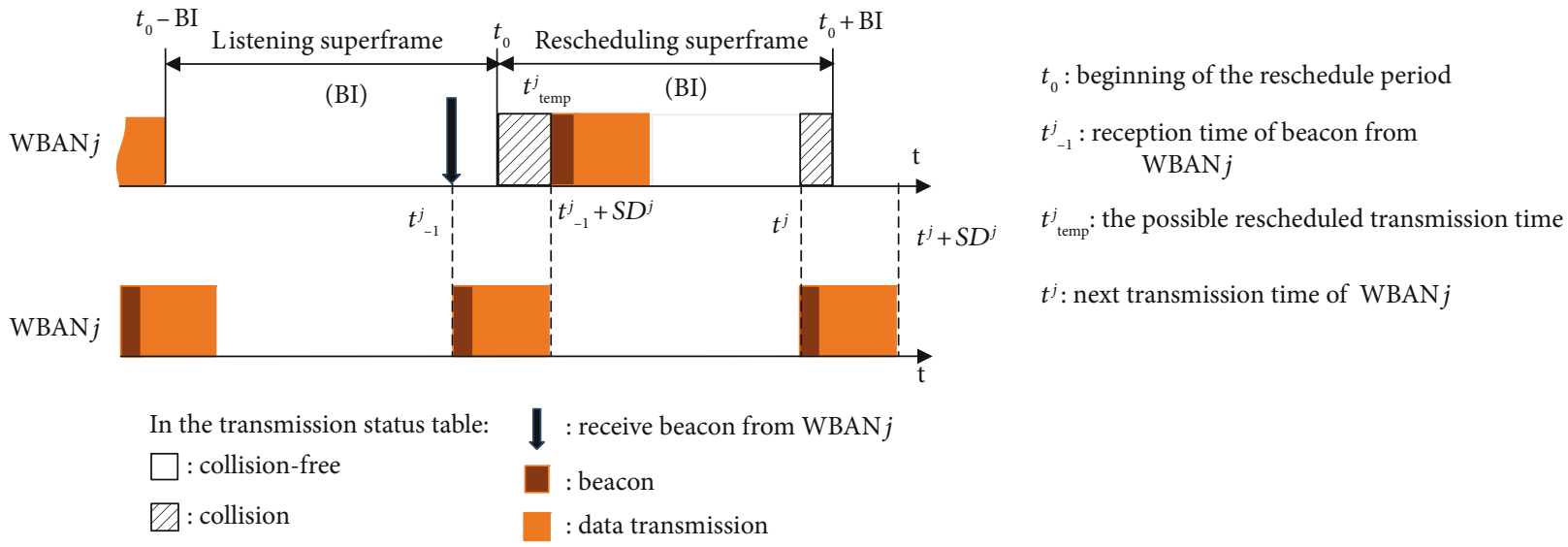

Figure 3: The transmission status table establishing procedure.

Figure 3 shows the process of establishing the neighbour transmission status table of network $i$, which is close to the body area of network $j$. After detecting the interference, the first step is to listen to the time of a superframe, and after receiving the beacon of $j$, it becomes known that the next transfer time $t_{0}$ will overlap, i.e., $t_{-1}^{j} \leq t_{0} \leq t_{-1}^{j}+$ $\mathrm{SD}^{j}$, and the overlap region $t^{k} \in\left(t_{0}, t_{-1}^{j}+\mathrm{SD}^{j}\right)$ represents the collision time. Then, the retransmission time is set to

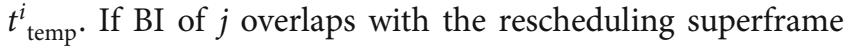
of $i$, i.e., $t_{0} \leq t^{j} \leq t_{0}+\mathrm{BI}$, we mark the timeslots $t^{k} \in\left(t^{j}\right.$, $\left.\min \left[t_{0}+\mathrm{BI}, t^{j}+\mathrm{SD}^{j}\right]\right)$ as collision regions. Other time gaps outside the collision regions are CFD.

When $n+1$ wireless body area networks are transmitting, it is necessary to avoid the collision between node $i$ and its $n$ neighbours. $\left(c^{i}, t^{i}\right)$ represents the new channel number and timeslot to be selected after rescheduling. In order to minimize the impact of interference, $T_{\text {delay }}$ must be as small as possible during data transmission.

$$
\left(c^{i}, t^{i}\right)=\underset{\left(c^{l}, t^{k}\right)}{\arg \min }\left(T_{\text {delay }}\left(c^{l}, t^{k}\right)\right) .
$$

We aim to find the corresponding empty time duration (Collision-Free Duration (CFD)) on the appropriate channel $c^{i}$ with the minimum possible $T_{\text {delay }}$ and find $t^{i}$ within CFD. Algorithm (OBTIM). The interference mitigation algorithm based on optimal backoff time is shown in Figure 4. It mainly includes the following phases:

(1) Initialization: when a WBANi experiences significant performance degradation, e.g., the coordinator identifies a significant decrease in throughput or packet reception rate, while the received signal strength does not obviously drop, the performance degradation is probably due to congestion instead of a bad channel. Start the interference mitigation initialization, and the coordinator enters the listening stage after the current active phase

(2) Neighbour discovery: the coordinator listens for the duration of a superframe length (BI) to collect its neighbours' information by decoding their beacon packets. It then uses the asynchronous neighbour discovery algorithm to record and build a neighbour table and eventually discover the neighbours

(3) Rescheduling: the coordinator performs the rescheduling algorithm to determine the possible rescheduling data transfer time $t_{\text {temp }}^{i}$. This step ensures that the overlapping transmission time is minimized without affecting other WBAN's ongoing transmissions. In the rescheduling phase, if the current channel is fully occupied, the rescheduling algorithm cannot obtain the rescheduling data transfer time $t_{\text {temp }}^{i}$ of the current channel. It will first find 


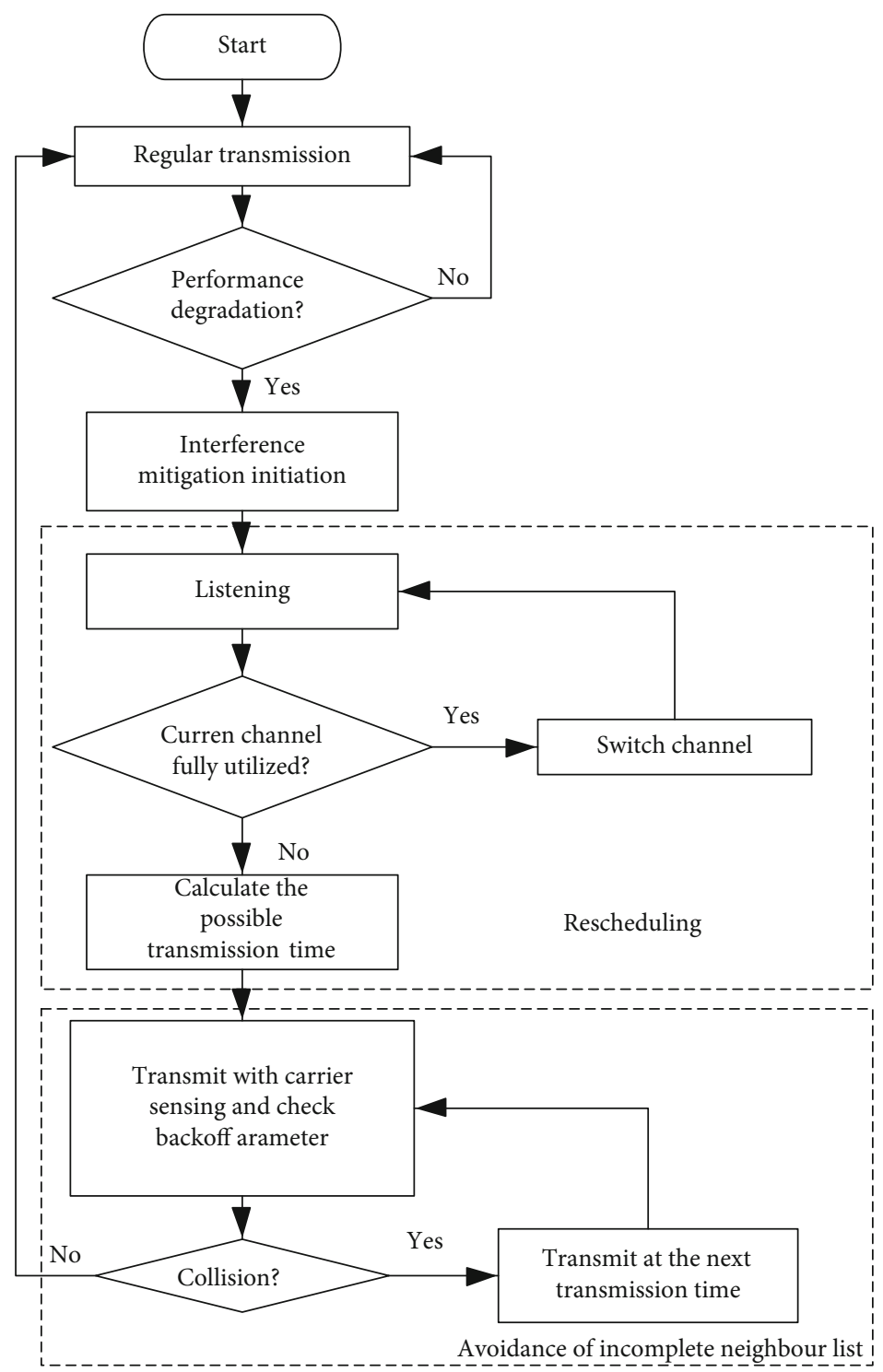

FIgURE 4: Flow chart of the internetwork interference mitigation method.

a channel that has timeslots. WBANi's coordinator broadcasts the idle channel to all WBANs, and the coordinator and WBAN $i$ then perform the rescheduling algorithm based on the idle channel too

(4) Data transfer: the coordinator notifies all WBANs of the new scheduling plan through the beacon, and after receiving the beacon information, the sensor nodes in WBAN $i$ periodically perform data transfer in the allocated timeslots until the next scheduled iteration begins. WBAN $i$ transmits at rescheduled transmission time $t_{\text {temp }}^{i}$ with carrier sensing and backoff, to avoid collision resulting from an incomplete neighbour list [32]

As shown in Figure 5, there exist WBAN1, WBAN2, WBAN3, and WBAN4 in a certain area. These WBANs data transfer in the same channel $C_{x}$. In the beginning, only WBAN1 is transmitting data, while WBAN2, WBAN3, and
WBAN4 move into the vicinity of WBAN1 in later points of time. After a period of listening, WBAN2, WBAN3, and WBAN4 are rescheduled to specific timeslots, in order to avoid conflict. Therefore, the transmission of WBAN1 is not affected. Imagine now that WBAN2 needs to transmit; it will first analyse its neighbour's beacon to calculate the occupancy and free time of WBAN1 on channel $C_{x}$. The rescheduling algorithm is then performed to calculate the rescheduled time $t_{\text {temp }}^{i}=t_{1}$, and then WBAN2 will start transmission from $t_{1}$. Similarly, after the arrival of WBAN4, it tries to decode the beacons of WBAN1, WBAN2, and WBAN3. In the listening phase of WBAN4, WBAN1 and WBAN2 have already noticed $C_{x}$ channel occupation information through their beacons. Note that WBAN3 is also in the listening stage. It has not been able to release the information of the channel occupancy through the beacon. Therefore, the beacons obtained by WBAN4 only have the occupancy information of WBAN1 and WBAN2, and the 


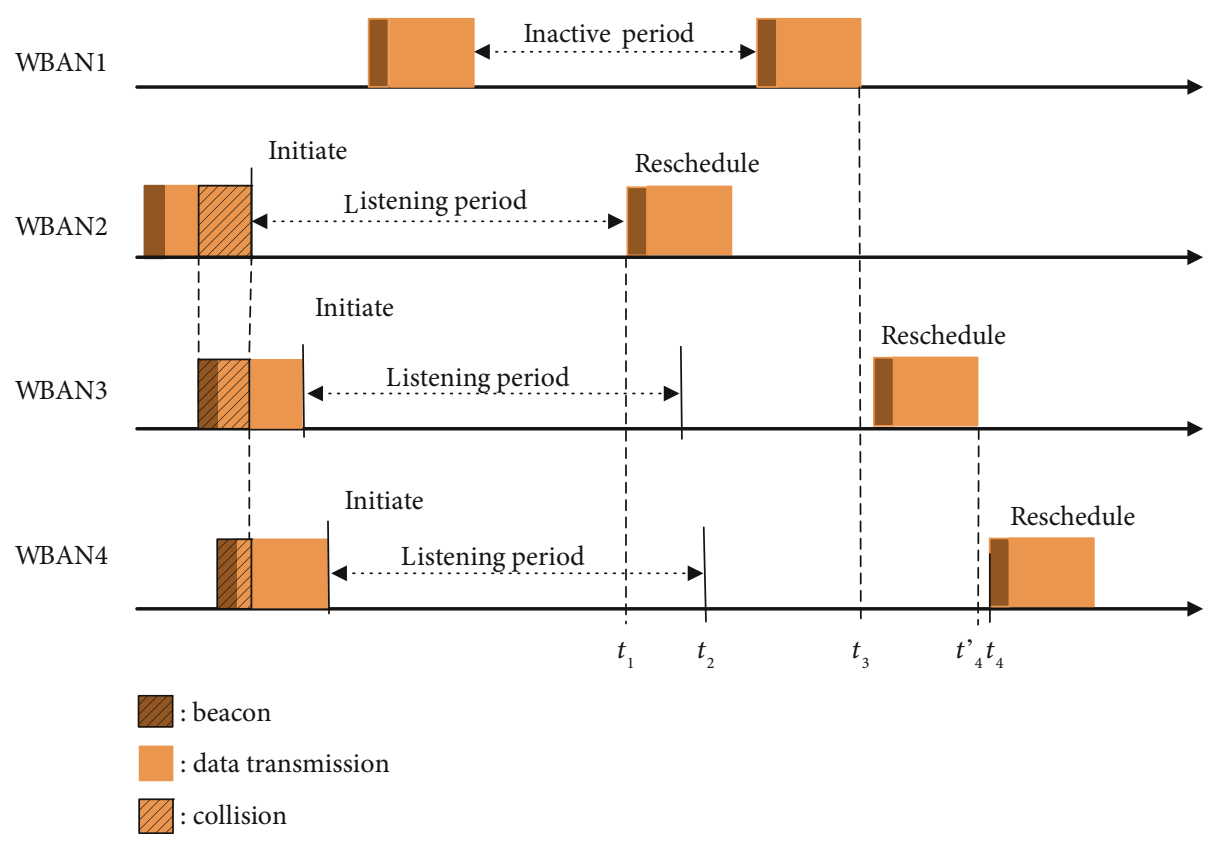

FIgURE 5: The implementation process of the internetwork interference mitigation method.

scheduling time of WBAN1 and WBAN2 on the channel $C_{x}$ is obtained. WBAN4 does not know the existence of WBAN3 in the listening phase of WBAN4. $t_{3}$ is the end time of WBAN1 scheduling, and $t_{2}$ is the end time of WBAN4 listening. WBAN4 knows from WBAN1's beacon that WBAN1 occupies channel $C_{x}$, which can only end at $t_{3}$, so it has to wait for the duration of $\left(t_{3}-t_{2}\right)$. WBAN3 in the meantime obtains the beacons to calculate the information for the channel $C_{x}$ which is the same as obtained by WBAN4. It is assumed that WBAN3 occupies channel $C_{x}$ before WBAN4, and transmission starts at $t_{3}$. When WBAN4 tries to transmit data through the channel at $t_{3}$, it will find that the channel has been occupied by WBAN3, so it has to wait for WBAN3 to complete transmission. It can be seen that $\left(t_{4}-t_{3}\right)$ is the time duration where WBAN4 and WBAN3 collide. At $t_{4}$, WBAN4 can transmit, but it keeps waiting for an extra moment because of the missing neighbour information from other nodes, then it starts formal transmission at $t_{4}$.

\subsection{The Asynchronous Neighbour Discovery Method} (ANDM). We make the following assumptions:

(1) Nodes cannot send or receive information simultaneously, but at any given time, one node performs either a sending or receiving function. To a node, the broadcasts transmitted from its neighbours are received error-free

(2) Each node is allocated a unique identifier, and it is aware of it

We assume there are $N$ neighbours in a particular area. We also assume a message $M$ is transmitted by the sender. We assume that message $M$ is successfully transmitted in time $T_{m}$. The receiver receives the message successfully if there are no other transmitters transmitting simultaneously within the specified distance. The message will not be received successfully if the transmissions collide. As we do not have global synchronization, so we include a preamble in message $M$. It is essential that the complete message is received.

Definition 1. Node $X$ is a neighbour of node $Y$, if $X$ can exceed $Y$ 's signal to noise ratio requirement.

Definition 2. Node $X$ discovers $Y$, if $X$ and $Y$ are neighbours and $X$ receives a message from $Y$ at least once. Node $X$ may discover $Y$, but $Y$ may not discover $X$.

Let us suppose there are $K$ nodes in total in an area, with unknown locations. We assume all sink nodes consist of the same number of timeslots, each having the same time duration, and each of them starts at random offset. There are two states $T$ (transmit) and $R$ (receive) to be chosen $\{T, R\}$ by each node in a timeslot. The probabilities are referred to as $p_{t}$ and $p_{r}$, respectively, with $p_{t}+p_{r}=1$. In each slot, the nodes choose their states to transmit or receive independently. The asynchronous neighbour discovery method mainly includes the following two steps:

Step 1. Determine the slot length.

Let $W$ be the number of copies of the message $M$ being sent, where $W$ is within a set of positive integers. In the case of slotted operations, $W>1$ is not necessary, but in unslotted operations, it is yet to be determined. We assume the transmitter is transmitting at its full capacity and the time required to switch between transmit and receive states is negligible. Let 
suppose time $T_{m}$ is required to transmit one copy of a message $M$, so to transmit $W$ copies the time required will be $W * T_{m}$, and the length of a single slot is $l=W * T_{m}$.

Step 2. Find the neighbours.

During the receiving state, the node turns on its receiver and decodes its input. This input is processed by assuming that the message is received error-free. After the transmitter identity in the message is known and found not within the node's neighbour list, it will be added, and if there is any new information, it will be updated at the receiver.

Let us analyse the Asynchronous Neighbour Discovery Method (ANDM) so as to facilitate further derivations.

Since there are a finite set of nodes and their neighbours are also finite, so there will be a finite set of relations. If nodes $X$ and $Y$ are neighbours, their relations $R(X, Y)$ and $R(Y, X)$ are both considered.

Let

$$
F(l)=E\left(\frac{R_{(X, Y)}(l)}{R_{(X, Y)}(r)}\right),
$$

where $R_{(X, Y)}(l)$ represents the number of relations discovered by ANDM in Step 1 and $R_{(X, Y)}(r)$ depicts the actual number of neighbour relations.

$E\left(\left(R_{(X, Y)}(l)\right) /\left(R_{(X, Y)}(r)\right)\right)$ depicts statistical expectations over the possible location of nodes and over random offset time.

As we have already assumed synchronization, so it is sufficient not to consider message repetition, so let $W=1$. Let us assume that Step 1 is fixed for neighbour discovery. This period consists of $S=\left[l / T_{m}\right]$ slots.

Let us also assume that $h$ is the number of successful receptions of a node in a single slot, while $p_{t}, W$, and $N$ are fixed. Therefore, $h$ is a random variable as $N-1$ states are unknown to us. It is therefore needed to calculate $E(h)$.

It is assumed that node $X$ has $N-1$ neighbours, since all $N$ nodes behave independently and each slot has a random offset. The slotted antilogarithm can be considered as the $N * S$ table with each cell having the Bernoulli random variable and $R$ and $T$ are transmitters and receivers among node $X$ neighbours. Since $h$ is the number of nodes heard by node $X$ in any given timeslot, it is always 0 or less than 1 . If $h$ increases to greater than 1 , it means there is a collision and $X$ will not hear any neighbour successfully.

In Table 1, it is shown that the synchronous neighbour discovery algorithm with $S$ timeslots and $N$ describes the state of a node. Each column represents the state of the system in a timeslot following binomial distribution. Assuming that node $X$ receives a message from one of its neighbours, if $X$ is in the receive state $R$, then only one of the neighbours is in the transmission state as in columns 5, 6, 8, and $S$.
TABLe 1: The timeslot and state of node $X$.

\begin{tabular}{lcccccccccc}
\hline Timeslot & 1 & 2 & 3 & 4 & 5 & 6 & 7 & 8 & $\cdots$ & $S$ \\
\hline Node $X$ & $R$ & $T$ & $R$ & $R$ & $R$ & $R$ & $R$ & $R$ & $\cdots$ & $R$ \\
Neighbour 1 & $R$ & $R$ & $T$ & $R$ & $R$ & $T$ & $R$ & $R$ & $\cdots$ & $R$ \\
Neighbour 2 & $R$ & $R$ & $R$ & $R$ & $R$ & $R$ & $R$ & $T$ & $\cdots$ & $R$ \\
Neighbour 3 & $R$ & $R$ & $T$ & $R$ & $R$ & $R$ & $R$ & $R$ & $\cdots$ & $R$ \\
$\vdots$ & $\vdots$ & & & & & & & & & \\
Neighbour $N-1$ & $R$ & $R$ & $R$ & $R$ & $T$ & $R$ & $R$ & $R$ & $\cdots$ & $T$ \\
\hline
\end{tabular}

Let $E(h)$ be the probability that $X$ hears a neighbour $Y$ in a single slot. Then

$$
\begin{aligned}
E(h) & =P_{r}(X \text { in state } R) P_{r}(l=1 \cdots S)=P_{R}{ }^{N-1} P_{T}\left(1-P_{T}\right)^{N-2} \\
& =(N-1) P_{T}\left(1-P_{T}\right)^{N-1} .
\end{aligned}
$$

As all the nodes are transmitting independently, the times that a neighbour is heard is uniformly distributed between 0 and $N-1$. So, the number of times that $X$ represents when a neighbour $Y$ is heard is $(E(h)) /(N-1)$. The performance of the algorithm is not dependent on a single node's fraction of neighbours discovered in the whole network.

$$
F=P_{r}(X \text { discovers } Y)=1-e^{-((S E(h)) /(N-1))} .
$$

Each timeslot is a trial in which neighbours might be heard when $(E(h)) /(N-1)$ is small and less possible to hear when the number of slots $S$ is large. Let $(E(h)) /(N-1)$ be the mean, then we can derive the possibility that $X$ can hear any neighbour that follows a Poisson distribution.

3.4. Rescheduling Algorithm. For an arbitrary WBAN $i$ assigned in channel $C_{x}$, the algorithm first finds out its neighbour list. For each neighbour in the neighbour list, $\mathrm{WBAN} j$ is found to have conflict time set $\alpha$ between WBAN $i$ and WBAN $j$, for the $n$ timeslots allocated by the system $\left(a_{1}, \cdots, a_{n}\right)$. If a timeslot $a_{k}$ falls in the conflict time set, then mark $a_{k}$ as the conflict timeslot. If there is a nonconflict time gap in $\left(a_{1}, \cdots, a_{n}\right)$ found, a nonconflict timeslot is allocated to WBAN $i$ for its next transmission. If the time gap in $\left(a_{1}, \cdots, a_{n}\right)$ consists of conflict timeslots only, it indicates that the current channel $C_{x}$ has been fully occupied. As a result, another channel $C_{y}$ will be temporarily assigned, and the above process will repeat to check if there exists any nonconflict timeslot [33].

3.4.1. Determine Conflict Timeslot Set $\alpha$. Set WBAN $i$ to start the rescheduling at $t_{0}$ time, then the listening period of WBAN $i$ is $\left(t_{0}-\mathrm{BI}\right)$ to $t_{0}$, and the rescheduling interval is $t_{0}$ to $\left(t_{0}+\mathrm{BI}\right)$. The current transmission time of WBAN $j$ is $t^{j}-1$, the next transmission moment is $t^{j}$, and the transmission duration is $\mathrm{SD}_{j}$. WBAN $i$ receives the beacon of WBAN $j$ at the $t^{j}-1$ moment of the 
listening stage $\left(t_{0}-\mathrm{BI}<t^{j}-1<t_{0}\right)$. It is obvious that, at first, if WBANi's rescheduling startup time $t_{0}$ falls within the current transmission time of $\mathrm{WBAN} j$, there will be conflict in $t_{-1}^{j} \leq t_{0} \leq t_{-1}^{j}+\mathrm{SD}^{j}$; and the second, if the next transmission time of WBAN $j$ falls within the rescheduling period of WBAN $i$, the conflict will occur in $t_{0} \leq t^{j} \leq t_{0}+\mathrm{BI}$. For each timeslot $a_{k}$, if the timeslot $a_{k} \in\left(\left(t_{0}, t_{-1}^{j}+\mathrm{SD}^{j}\right) \cup\left(t^{j}, \min \left(t_{0}+\mathrm{BI}, t^{j}+\mathrm{SD}^{j}\right)\right)\right)$, then the timeslot $a_{k}$ can be marked as a conflict timeslot.

\subsubsection{A Method of Backoff Time Optimization Based on} Linear Programming. As mentioned in Section 3.1, each scheduling operation includes a data transfer delay $T_{\text {delay, }}$, and in order to maximize throughput, $T_{\text {delay }}$ must be minimal for data transfer delay. Therefore, as for WBAN $i$, given minimal data transmission delay $T_{\text {delay }}$, the purpose of this work is to find out one timeslot in noncompetitive time CF $\mathrm{D}_{i}$ (collision-free duration) in channel $C_{x}$. The beginning time of $\mathrm{CFD}_{i}$ is $t_{i}$, and SD is the data transmission duration and $\mathrm{SD}$ is a fixed value.

We make an assumption that all the WBANs in the system have the same transmission time BI and the same SD, and the transmission order in the channel is the front one next to the next; thus, the channel capacity in $C_{x}$ is as follows:

$$
N_{\text {WBAN }}\left(C_{x}\right)=\left\lfloor\frac{\mathrm{BI}}{\mathrm{SD}}\right\rfloor \text {. }
$$

[- is the integral operation; note that $N_{\text {WBAN }}\left(C_{x}\right)$ is actually the inverse of SD/BI. In the case of multiple $m$ channels available in the system, then the number of WBAN can reach

$$
N_{\max }=\sum_{c=1}^{m}\left\lfloor\frac{\mathrm{BI}}{\mathrm{SD}}\right\rfloor \text {. }
$$

With regards to the transmission delay $T_{\text {delay }}$, it can be defined as the time period from the source transmission time to the rescheduling time, which includes the backoff time $T_{\text {backoff }}$ and the work delay $T_{\text {work }}$. $T_{\text {backoff }}$ represents backing off for a period of time before sending the rescheduling beacon. In order to prevent an incomplete neighbour list or hidden terminals, the system sets up a fallback strategy deliberately. $T_{\text {work }}$ indicates the delay introduced in the scheduling process due to some factors, one being in the transmission stage; in order to avoid overlapping WBAN with neighbours, it needs to delay for a period of time. Another could be when a node fails to compete with other WBAN channels; it needs to wait for the next timeslot. Obviously, the node will scan $n$ channels to find suitable scheduling in its current channel. Before finding a suitable scheduling scheme, the transmission delay $\Phi\left(N_{\max }\right)$ must meet the following condition:

$$
\left\{\begin{array}{l}
T_{\text {delay }}<(\mathrm{SD}+\mathrm{BI}), \\
T_{\text {delay }}=T_{\text {backoff }}+(n-1) * T_{\text {work }}
\end{array}\right.
$$

Each WBAN has three states, namely, the interference transmission state, the execution state, and the normal transmission state. WBAN is converted in these three states accordingly. When interference occurs, WBAN transmission could be affected severely, which will start the rescheduling algorithm to find a suitable channel; after interference reduces, the system is back to normal operations until the next interference comes.

Assuming the system has already run the $K$ iterations, the delay time caused by the algorithm is $T_{\text {delay }}(K)$ and the regular transmission time is $T_{\text {regular }}(K)$. Then, the time of the algorithm is $T_{\text {time }}$ :

$$
T_{\text {time }}=T_{\text {delay }}(K)+T_{\text {regular }}(K) \text {. }
$$

The throughput $\Psi(K)$ of a regular transmission is related to the number of WBAN and can be expressed as a function of the number of WBAN as follows:

$$
\Psi(K)=\frac{T_{\text {regular }}(K)}{T_{\text {delay }}(K)+T_{\text {regular }}(K)} \Phi\left(N_{\text {max }}\right)
$$

To avoid a conflict, each scheduling operation includes a data transfer delay $T_{\text {delay; }}$ whether $T_{\text {delay }}$ is appropriate or not, it will affect the throughput of the system. The solution of the above problem can be explained as searching for a suitable backoff time $T_{\text {backoff }}$ to maximize throughput $\Psi(k)$ which can be abstractly posed as a linear programming problem, under the agreed condition $T_{\text {delay }}=T_{\text {work }}+T_{\text {backoff }}$ and $T_{\text {delay }}<(\mathrm{SD}+\mathrm{BI})$. The mathematical model can then be rewritten as follows to maximize the objective function:

$$
\left\{\begin{array}{l}
\max \int_{0}^{K} \Psi(k) d_{k} \\
T_{\text {delay }}=T_{\text {work }}+T_{\text {backoff }} \\
T_{\text {delay }}<(\mathrm{SD}+\mathrm{BI}) .
\end{array}\right.
$$
have

Substituting formulas (8) and (9) into equation (10), we

$$
\left\{\begin{array}{l}
\max \int_{0}^{K} \frac{T_{\text {regular }}(k) \Phi\left(N_{\text {max }}\right)}{T_{\text {backoff }}(k)+T_{\text {work }}(k)+T_{\text {regular }}(k)} d_{k}, \\
\int_{0}^{k}\left(T_{\text {backoff }}(k)+T_{\text {work }}(k)\right) d_{k}<k \cdot(\mathrm{SD}+\mathrm{BI}) .
\end{array}\right.
$$

It can be seen from formula (9) that $\Psi(k)$ decreases when $K$ increases; therefore, it can be considered strictly concave. $K$ is a certain positive time, and $T_{\text {work }}(k) \geq 0$, then the constraint space $Z$ corresponds to the inequality:

$$
\int_{0}^{k} T_{\text {backoff }}(k) d_{k} \leq k \cdot(\mathrm{SD}+\mathrm{BI}) .
$$


Input: Neighbour list of WBAN $i\left\{\Omega^{i},\left(S D^{j}, B I^{j}, C^{j}, t^{j}\right) \mid 1 \leq j \leq N j \neq i\right\}$

Output: Collision-free transmission opportunity $t_{\text {temp }}^{i}$

1. At the end of the listening period WBAN $i$ performs the following:

2. Allocate $n$ slots $\left(a_{1}, \ldots, a_{n}\right)$

3. for WNAN $j$ the neighbour list $\Omega^{i}$ do

4. if there is transmission going on, start rescheduling at $t_{0}$,

$$
t_{-1}^{j} \leq t_{0} \leq t_{-1}^{j}+S D^{j} \text { then }
$$

5. $a_{k} \in\left(t_{0}, t_{-1}^{j}+S D^{j}\right) \longrightarrow$ collision occurs

6. end if

7. if the next transmission of the WBAN $j$ is in the rescheduled transmission frame of WNAN $i$. i.e., $t_{0} \leq t^{j} \leq t_{0}+B I$ then

8. $a_{k} \in\left(t^{j} \min \left[t_{0}+B I, t^{j}+S D^{j}\right]\right) \longrightarrow$ collision occurs

9. end if

10. end for

11. for $a_{k}$ in the rescheduling superframe do

12. The $T_{\text {backoff }}$ is calculated according to formula (18), and then according to formula (7), the $T_{\text {delay }}$ is obtained by $T_{\text {backoff }}$.

13. if there is a collision-free time duration starting with $a_{k}$ for the transmission of WBAN $i$ then

14. Assign $a_{k}$ to $t_{\text {temp }}^{i}(q)$;

15. Keep looking for the next slot:

16. $q \longleftarrow q+1$;

17. $k \longleftarrow k+1$;

18. end if

19. end for

20. if collision-free duration is not found, $q=0$ then

21. Switch channel;

end if

Algorithm 1: Rescheduling algorithm.

The Lagrangian of this question is as follows:

$$
\begin{aligned}
L\left(T_{\text {backoff }}, \lambda\right)= & \int_{0}^{K} \frac{T_{\text {regular }}(k) \Phi\left(N_{\text {max }}\right)}{T_{\text {backoff }}(k)+T_{\text {work }}(k)+T_{\text {regular }}(k)} d_{k} \\
& -\lambda\left(\int_{0}^{K} T_{\text {backoff }}(k) d_{k}-k \cdot(\mathrm{BI}+\mathrm{SD})\right) .
\end{aligned}
$$

In this situation, the optimal solution $T_{\text {backoff }}^{0}(k) \geq 0$ can be obtained, and the corresponding $\lambda_{0}$ satisfies the following formula:

$$
\min _{\lambda \geq 0} \max _{T_{\text {backoff }} \geq 0} L\left(T_{\text {backoff }}, \lambda\right)=L\left(T_{\text {backoff }}^{0}, \lambda_{0}\right) .
$$

Given that $\Psi(k)$ is a concave function and according to the necessity theorem of differentiable conditions, we solve for $\max _{T_{\text {backoff }} \geq 0} L\left(T_{\text {backoff }}, \lambda\right)$, which is equivalent to the following problems:

$$
\int_{0}^{K}\left(\frac{T_{\text {regular }}(k) \Phi\left(N_{\text {max }}\right)}{T_{\text {backoff }}^{0}(k)+T_{\text {work }}(k)+T_{\text {regular }}(k)}-\lambda_{0}\right) T_{\text {backoff }} f(k) d_{k} \leq 0
$$

or

$\int_{0}^{K}\left(\frac{T_{\text {regular }}(k) \Phi\left(N_{\text {max }}\right)}{T_{\text {backoff }}^{0}(k)+T_{\text {work }}(k)+T_{\text {regular }}(k)}-\lambda_{0}\right) T_{\text {backoff }} f(k) d_{k}=0$.

Apparently $\lambda_{0}>0$, so this is equivalent to solving $T_{\text {backoff }}^{0}(k), \lambda_{0}$, and $\mu(k)$ in formula (17) in the condition of $k \in[0, K]$.

$$
\left\{\begin{array}{l}
T_{\text {backoff }}^{0}(k) \geq 0, \lambda_{0}>0, \mu(k) \geq 0, \\
\mu(k) T_{\text {backoff }}^{0}(k)=0, \\
\int_{0}^{K} T_{\text {backoff }}^{0}(k) d_{k}=k \cdot(\mathrm{BI}+\mathrm{SD}), \\
\frac{T_{\text {regular }}(k) \Phi\left(N_{\text {max }}\right)}{T_{\text {backoff }}^{0}(k)+T_{\text {work }}(k)+T_{\text {regular }}(k)}-\lambda_{0}+\mu(k)=0 .
\end{array}\right.
$$

Assume $k_{0}$ is the maximum time point of system throughput, and we can obtain by formula (17) the following:

$$
T_{\text {backoff }}^{0}(k)=\frac{T_{\text {regular }}\left(k_{0}\right) \Phi\left(N_{\text {max }}\right)}{1+T_{\text {work }}\left(k_{0}\right)+T_{\text {regular }}\left(k_{0}\right)} .
$$

3.4.3. Implementation of Rescheduling Algorithm. In the rescheduling phase, if the current channel is fully occupied, 
TABLE 2: The simulation parameter settings.

\begin{tabular}{lccccccccc}
\hline $\begin{array}{l}\text { Parameter } \\
\text { name }\end{array}$ & $\begin{array}{c}\text { Transmission } \\
\text { power }(\mathrm{dBm})\end{array}$ & $\begin{array}{c}\text { Mobile speed } \\
\text { interval }(\mathrm{m} / \mathrm{s})\end{array}$ & $\begin{array}{c}\text { Motion } \\
\text { interval } \\
(\mathrm{s})\end{array}$ & $\begin{array}{c}\text { Pause } \\
\text { interval } \\
(\mathrm{s})\end{array}$ & $\begin{array}{c}\text { Direction } \\
\text { interval } \\
(\text { degree })\end{array}$ & $\begin{array}{c}\text { Simulation } \\
\text { duration }(\mathrm{s})\end{array}$ & $\begin{array}{c}\text { Superframe } \\
\text { duration (SD) } \\
(\mathrm{s})\end{array}$ & $\begin{array}{c}\text { Maximum } \\
\text { tolerable } \\
\text { delay(s) }\end{array}$ & $\begin{array}{c}\text { Content } \\
\text { window } \\
(\mathrm{CW})\end{array}$ \\
\hline Value & -10 & $(0.2,2.2)$ & $(2,6)$ & $(0,6)$ & $(-180,180)$ & 1000 & 0.1 & 0.15 & $(1,8)$ \\
\hline
\end{tabular}

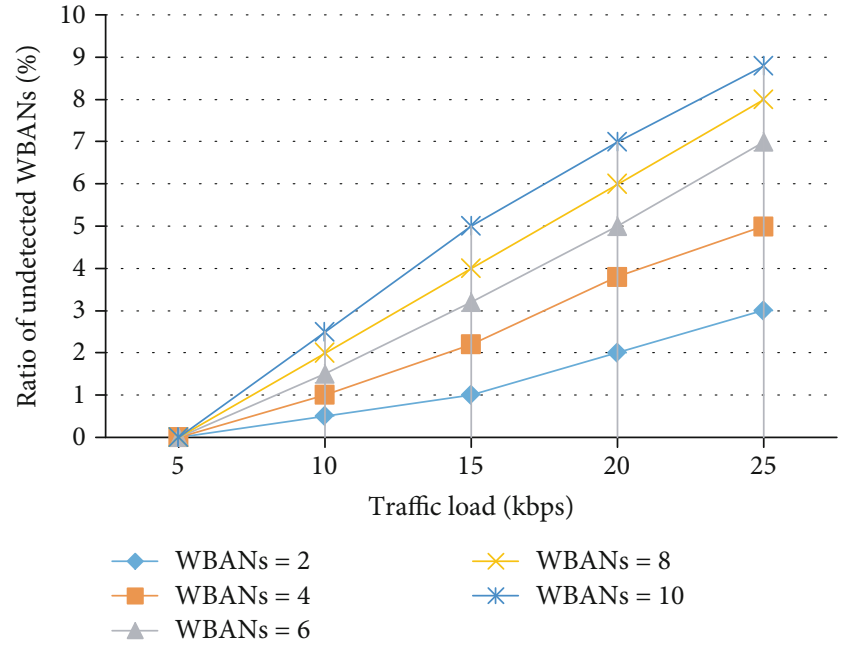

Figure 6: The ratio of undiscovered WBANs to neighbouring WBANs.

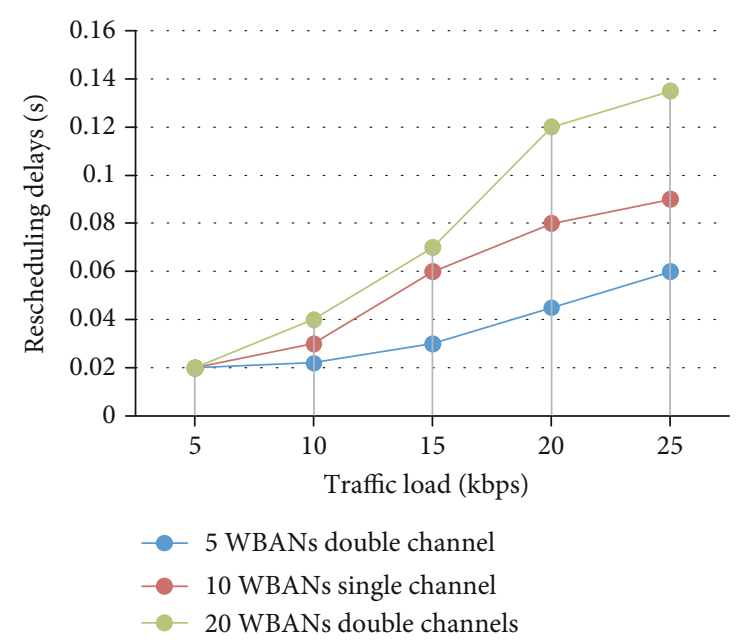

FIgURE 7: Rescheduling delay for three different scenarios.

the rescheduling algorithm cannot obtain the rescheduling data transfer time of the current channel. It will first find a channel that has timeslots. WBANi's coordinator broadcasts the idle channel to all WBANs, and the coordinator and WBAN $i$ then perform the rescheduling algorithm based on the idle channel too. Algorithm 1 shows the rescheduling algorithm.

\section{Simulations and Results}

We assume that each WBAN contains a coordinator and a sensor node. The physical layer parameters were set accord-

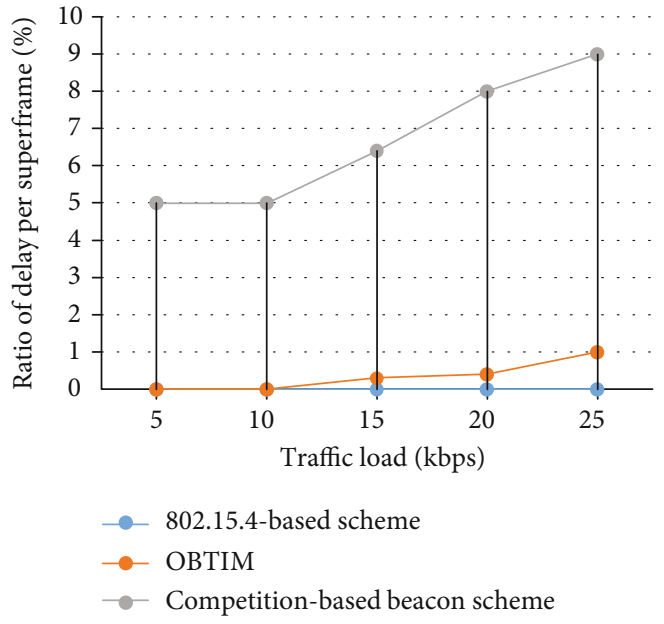

FIgURE 8: The ratio of average delay to the beacon interval.

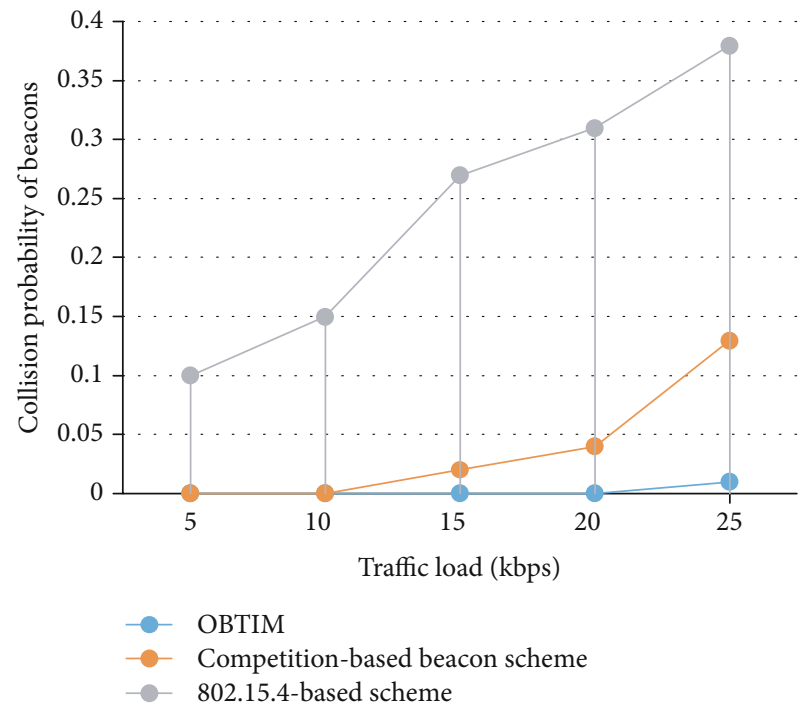

Figure 9: Collision probability of beacon comparison.

ing to the standard of IEEE802.15.4. The interbody path loss model is considered, and the path loss exponent is 2.4 with a shadowing standard deviation of $6 \mathrm{~dB}$. We choose superframe length $\mathrm{SD}=0.1 \mathrm{~s}$ and radio data rate as $250 \mathrm{kbps}$, considering that the typical medical EEG and ECG applications have reached the data rate of $5 \mathrm{kbps}$ and the temperature, respiratory, and pulse sensors typically have a data rate of $1 \mathrm{kbps}$. To make use of all these combined sensors, traffic load per WBAN changes from 5 to $25 \mathrm{kbps}$. Traffic for all WBANs is set the same for the sake of simplicity, as shown in Table 2 as a specified scenario. 


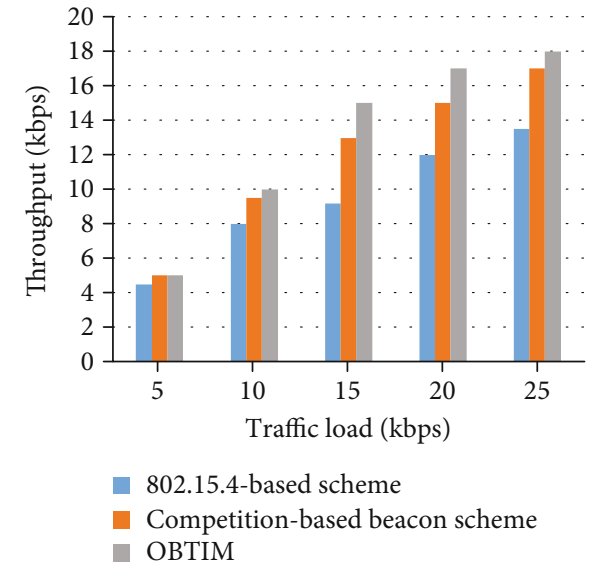

(a) 5 WBANs in a single channel

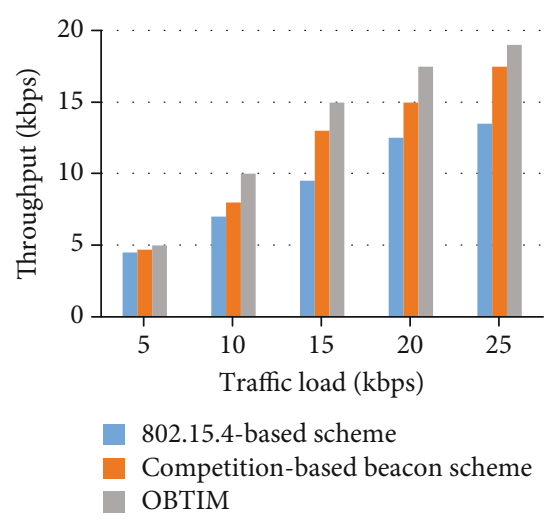

(b) 10 WBANs in a single channel

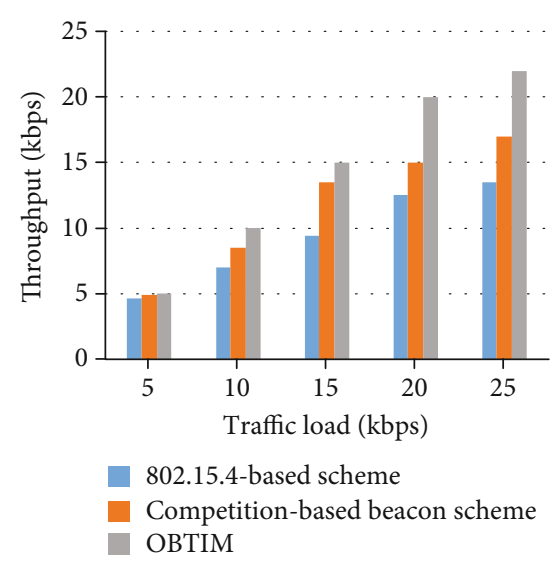

(c) 20 WBANs in double channels

FIgURE 10: Throughput versus traffic load for three scenarios.

We assume that each WBAN user occupies an area of $5 \sim 10$ square meters, with 5 randomly moving users in the $30 \times 30 \mathrm{~m}$ space. Later, we also simulate the cases of 10 and 20 users. We consider the random way point model [34, 35] for movement.

We can find that with the increase in the number of neighbours and the traffic load of WBAN, the interference will increase. This will lead to more beacon loss. It can be seen from Figure 6 that even in the worst case (10-WBAN aggregation and $25 \mathrm{kbps}$ traffic each), our proposed method can ensure that the neighbour table catches more than $91 \%$ of the neighbour wireless body area network information. Therefore, as for rescheduling, the neighbour list table proves to be a useful tool that can provide enough information to guarantee reliable transmission.

Figure 7 depicts the average rescheduling delay of the proposed method for three cases: 5 WBANs single channel; 10 WBANs single channel and 20 WBANs double channels. The results show that time delay is less than $0.09 \mathrm{~s}$ in the single-channel case and proves that it is always less than $(\mathrm{SD}+\mathrm{BI})$ and WBAN can find an appropriate scheduling scheme in the current channel. In the case of double channels, the delay increases but is still less than $(2 \mathrm{BI}+\mathrm{SD})$. With the increase in communication traffic, it becomes more difficult to find an appropriate rescheduling, but the maximum delay of $0.135 \mathrm{~s}$ is still suitable for most applications.

Figure 8 shows that the competition-based beacon scheme requires multiple carrier sensing for each transmission. In such a scheme, when the wireless body number increases due to the lack of an effective means of channel switching, channel congestion will lead to a longer backoff time and the delay will increase significantly. The OBTIM only performs rescheduling when the interference condition is detected, and it reduces the number of collisions, which leads to minimized delay.

The beacon collision probability in three schemes is compared in Figure 9. By using the rescheduling strategy, the collision probability in the OBTIM is the lowest. In the 802.15.4 basic scheme, since the transmissions are at fixed schedules, the collisions are highest. For the competition-based beacon scheme, WBAN competes with all neighbouring
WBANs to access the channel; therefore, collision happens much more often than that of the interference mitigation scheme (the one we proposed). As most of the data in the wireless body area network have periodicity, once conflict occurs, each frame will conflict in the whole interference period.

Figure 10 depicts that in terms of data throughput for three different WBANs and channel numbers, the OBTIM outperforms by up to $30 \%$ and $18 \%$ compared to the 802.15.4 basic scheme and the competition-based beacon scheme, respectively. (a) and (b) also depict that if WBAN increases from 5 to 10 , the performance of the 802.15 .4 basic scheme decreases significantly. Moreover, the time delay of the competition-based beacon-enabled scheme is larger, and collisions are more often. Although the average number of WBANs in each channel is the same, the throughput of the OBTIM is better than that of the single channel in the dualchannel scenario. This is because other schemes can only compete within the current channel, resulting in a sustained drop in throughput.

As shown in Figure 11, because the OBTIM can avoid collisions effectively, it, therefore, alleviates the interference and helps reduce the retransmission. The average energy consumption of the interference mitigation scheme for the successful transmission of a data packet is significantly lower than the other two schemes. Energy consumption of the proposed scheme per packet is $20 \%$ lower than that of the competition-based beacon scheme and 16\% than that of the 802.15.4 basic scheme. When the communication load increases, the latter two schemes have to consume a large amount of energy for multiple carrier interception.

\section{Conclusions}

In this paper, a distributed internetwork interference mitigation scheme is proposed for body area sensor networks. The scheme takes into account entirely the low utilization rate of the network channel and adopts the scheduling strategy based on the optimal backoff time, so that the transmission time and channel can be selected reasonably when the network is disturbed: (1) when the channel utilization is low, 


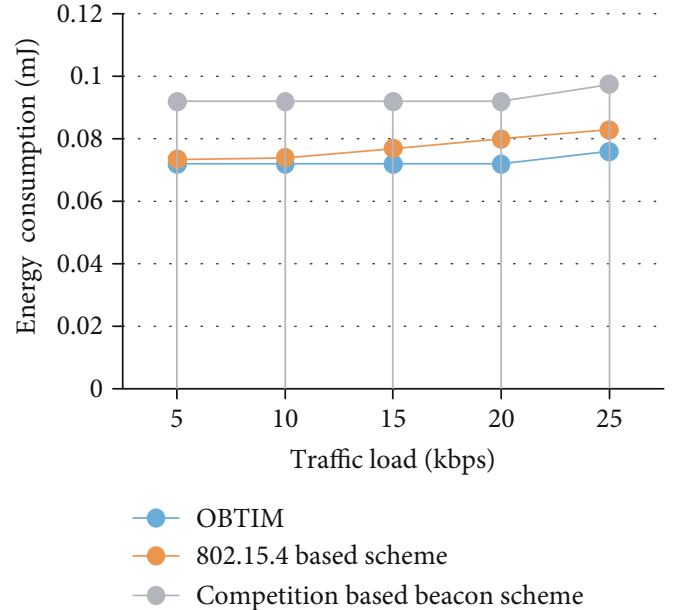

FIGURE 11: Comparison of per-packet energy consumption (10 WBANs per single channel).

the body area network is rescheduled by the coordinator on a free timeslot, and (2) when the current channel is fully occupied, the coordinator switches the channel in time. Simulation results show that the proposed approach of OBTIM outperforms the 802.15.4 base scheme and the competitionbased signal scheme in producing fewer collisions, higher throughput, and lower energy consumption. Furthermore, when the density of the WBANs increases, the proposed method enables the channel switch to make reasonable adjustments to the transmission condition, to ensure the reliability of data transmission.

\section{Data Availability}

The data used to support the findings of this study are available from the corresponding author upon request.

\section{Conflicts of Interest}

The authors declare that there is no conflict of interest regarding the publication of this paper.

\section{Acknowledgments}

This work was supported by the National Natural Science Foundation of China (No. 61971248), the International Science and Technology Cooperation Programme (No. 2016D10008), the Ningbo Key Science and Technology Plan (2025) Project (2018B10075, 2019B10125, and 2019B10028), and the Key Science and Technology Projects of Zhejiang Province (2020C03064).

\section{References}

[1] B. Latré, B. Braem, I. Moerman, C. Blondia, and P. Demeester, "A survey on wireless body area networks," Wireless Networks, vol. 17, no. 1, article 252, pp. 1-18, 2011.

[2] H. B. Lim, D. Baumann, and E. P. Li, "A human body model for efficient numerical characterization of UWB signal propagation in wireless body area networks," IEEE Transactions on Biomedical Engineering, vol. 58, no. 3, pp. 689-697, 2011.
[3] X. Liang, X. Li, Q. Shen et al., "Exploiting prediction to enable secure and reliable routing in wireless body area networks," 2012 Proceedings IEEE INFOCOM, 2012, pp. 388-396, Orlando, FL, USA, March 2012.

[4] Z. Wang, M. Jiang, Y. Hu, and H. Li, "An incremental learning method based on probabilistic neural networks and adjustable fuzzy clustering for human activity recognition by using wearable sensors," IEEE Transactions on Information Technology in Biomedicine, vol. 16, no. 4, pp. 691-699, 2012.

[5] A. Natarajan, M. Motani, B. de Silva, K.-K. Yap, and K. C. Chua, "Investigating network architectures for body sensor networks," in Proceedings of the 1st ACM SIGMOBILE international workshop on Systems and networking support for healthcare and assisted living environments, pp. 19-24, San Juan, Puerto Rico, June 2007.

[6] W. Sun, Y. Ge, and W. C. Wong, "A lightweight distributed scheme for mitigating inter-user interference in body sensor networks," Computer Networks, vol. 57, no. 18, pp. 38853896, 2013.

[7] M. Chen, S. Gonzalez, A. Vasilakos, H. Cao, and V. C. M. Leung, "Body area networks: a survey," Mobile networks and applications, vol. 16, no. 2, pp. 171-193, 2011.

[8] H. Wang, Z. Zhang, X. Lin, and H. Fang, "Socialized WBANs in mobile sensing environments," IEEE Network, vol. 28, no. 5, pp. 91-95, 2014.

[9] Y. Xiao, X. Shan, and Y. Ren, "Game theory models for IEEE 802.11 DCF in wireless ad hoc networks," IEEE Communications Magazine, vol. 43, no. 3, pp. S22-S26, 2005.

[10] M. A. Ergin, K. Ramachandran, and M. Gruteser, "Understanding the effect of access point density on wireless LAN performance," in Proceedings of the 13th annual ACM international conference on Mobile computing and networking, pp. 350-353, Montreal, Quebec, Canada, September 2007.

[11] T. Wu and S. Biswas, "Reducing inter-cluster TDMA interference by adaptive MAC allocation in sensor networks," in Sixth IEEE International Symposium on a World of Wireless Mobile and Multimedia Networks, pp. 507-511, Taormina-Giardini Naxos, Italy, June 2005.

[12] T. Wu, F. Wu, J. M. Redouté, and M. R. Yuce, "An autonomous wireless body area network implementation towards IoT connected healthcare applications," Ieee Access, vol. 5, pp. 11413-11422, 2017.

[13] M. Waheed, R. Ahmad, W. Ahmed, M. Drieberg, and M. Alam, "Towards efficient wireless body area network using two-way relay cooperation," Sensors, vol. 18, no. 2, p. 565, 2018.

[14] S. Kim, S. Kim, J. W. Kim, and D. S. Eom, "Flexible beacon scheduling scheme for interference mitigation in body sensor networks," in 2012 9th Annual IEEE Communications Society Conference on Sensor, Mesh and Ad Hoc Communications and Networks (SECON), pp. 157-164, Seoul, South Korea, June 2012.

[15] A. F. Molisch, P. Orlik, Z. Sahinoglu, and J. Zhang, "UWBbased sensor networks and the IEEE 802.15.4a standard-a tutorial," in 2006 First International Conference on Communications and Networking in China, pp. 1-6, Beijing, China, October 2006.

[16] S. González-Valenzuela, M. Chen, and V. C. Leung, "Mobility support for health monitoring at home using wearable sensors," IEEE Transactions on Information Technology in Biomedicine, vol. 15, no. 4, pp. 539-549, 2011. 
[17] W. Zou, F. Kang, G. Du, and G. Zhang, "Physical layer proposal design and interference analysis based on chinese medical band," Journal of Electronics and Information Technology, vol. 37, no. 2, pp. 429-434, 2015.

[18] G. Wu, J. Ren, F. Xia, L. Yao, and Z. Xu, “DISG: decentralized inter-user interference suppression in body sensor networks with non-cooperative game," in 2010 7th International Conference on Ubiquitous Intelligence \& Computing and 7th International Conference on Autonomic \& Trusted Computing, pp. 256-261, Xian, Shaanxi, China, October 2010.

[19] J. W. Kim, K. Hur, and S. R. Lee, "A robust beacon scheduling scheme for coexistence between UWB based WBAN and WiMedia networks," Wireless Personal Communications, vol. 80, no. 1, pp. 303-319, 2015.

[20] S. Movassaghi, A. Majidi, A. Jamalipour, D. Smith, and M. Abolhasan, "Enabling interference-aware and energyefficient coexistence of multiple wireless body area networks with unknown dynamics," IEEE Access, vol. 4, pp. 29352951, 2016.

[21] M. Roy, C. Chowdhury, and N. Aslam, "Designing an energy efficient WBAN routing protocol," in 2017 9th International Conference on Communication Systems and Networks (COMSNETS), Bangalore, India, January 2017.

[22] Q. Li, Wireless Body Domain Network Collaboration Network Relay Selection and Power Allocation in [D], Yunnan University, 2014.

[23] T. T. T. Le and S. Moh, "An interference-aware traffic-prioritybased link scheduling algorithm for interference mitigation in multiple wireless body area networks," Sensors, vol. 16, no. 12, p. 2190, 2016.

[24] L. Yu, Research on Data Fusion Green Model and Transmission Scheduling Algorithm in Wireless Body Domain Network, China Agricultural University, 2016.

[25] T. Le and S. Moh, "Hybrid multi-channel MAC protocol for WBANs with inter-WBAN interference mitigation," Sensors, vol. 18 , no. 5 , p. $1373,2018$.

[26] L. Li, Y. Xu, Z. Zhang, J. Yin, W. Chen, and Z. Han, “A prediction-based charging policy and interference mitigation approach in the wireless powered Internet of Things," IEEE Journal on Selected Areas in Communications, vol. 37, no. 2, pp. 439-451, 2019.

[27] Y. Tian, "A non-orthogonal multiple access and interference mitigation combined strategy in multi-user networks," Wireless Personal Communications, vol. 104, no. 1, article 6011, pp. 111-128, 2019.

[28] H. Wang, Key Technologies of Collaborative Communication, Beijing University of Posts and Telecommunications, 2015.

[29] S. Minlei, Key Technology Research on the MAC Layer in the Wireless Domain Network for Medical Monitoring, Shandong University, 2016.

[30] C. Li, B. Zhang, X. Yuan, S. Ullah, and A. V. Vasilakos, "MCMAC: a multi-channel based MAC scheme for interference mitigation in WBANs," Wireless Networks, vol. 24, no. 3, pp. 719-733, 2018.

[31] J. Shao, "A CSMA/CA based MAC layer solution for interWBAN interference and starvation," Master's thesis, University of Waterloo, 2015.

[32] R. W. Koller, L. A. Ricardez-Sandoval, and L. T. Biegler, "Stochastic back-off algorithm for simultaneous design, control, and scheduling of multiproduct systems under uncertainty," AICHE Journal, vol. 64, no. 7, pp. 2379-2389, 2018.
[33] H. Moungla, A. Jarray, A. Karmouch, and A. Mehaoua, "Cost-effective reliability-and energy-based intra-WBAN interference mitigation," in 2014 IEEE Global Communications Conference, pp. 2399-2404, December 2014.

[34] S. M. Mousavi, H. R. Rabiee, M. Moshref, and A. Dabirmoghaddam, "MobiSim: a framework for simulation of mobility models in mobile ad-hoc networks," in Third IEEE International Conference on Wireless and Mobile Computing, Networking and Communications (WiMob 2007), pp. 82-82, White Plains, NY, USA, October 2007.

[35] A. Ribeiro and R. Sofia, A survey on mobility models for wireless networks, SITI, University Lusófona, 2011. 